Cite this: Nanoscale, 2014, 6, 6065

\title{
Towards graphene bromide: bromination of graphite oxide $\uparrow$
}

\author{
O. Jankovský, ${ }^{a}$ P. Šimek, ${ }^{a}$ K. Klimová, ${ }^{a}$ D. Sedmidubský, ${ }^{a}$ S. Matějková, ${ }^{b}$ M. Pumera \\ and Z. Sofer ${ }^{\star a}$
}

Halogenated graphene derivatives are interesting for their outstanding physical and chemical properties. In this paper, we present various methods for the synthesis of brominated graphene derivatives by the bromination of graphite oxides. Graphite oxides, prepared according to either the Hummers or Hofmann method, were brominated using bromine or hydrobromic acid under reflux or in an autoclave at elevated temperatures and pressures. The influence of both graphite oxide precursors on the resulting brominated graphenes was investigated by characterization of the graphenes, which was carried out using various techniques, including SEM, SEM-EDS, high-resolution XPS, FTIR, STA and Raman spectroscopy. In addition, the resistivity of the brominated graphenes was measured and the electrochemical properties were investigated by cyclic voltammetry. Although the brominated graphenes

Received 2nd March 2014

Accepted 25th March 2014

DOI: $10.1039 / c 4 n r 01154 f$

www.rsc.org/nanoscale were structurally similar, they had remarkably different bromine concentrations. The most highly brominated graphene (bromine concentration above $26 \mathrm{wt} \%$ ) exhibited a C/O ratio above 44 and partial hydrogenation. Brominated graphenes with such properties could be used for reversible bromine storage or as a starting material for further chemical modifications.

\section{Introduction}

Since its discovery in 2004, graphene has attracted widespread interest because of its unique electrical, optical and mechanical properties and broad range of potential applications. ${ }^{1}$ The outstanding properties of this $2 \mathrm{D}$ carbon-based material can be applied to microelectronic and optoelectronic devices, ${ }^{2}$ electrochemical and biochemical sensors ${ }^{3}$ and energy storage materials. ${ }^{4}$ Perhaps even more importantly, the properties of graphene can be tailored through chemical modifications, such as hydrogenation ${ }^{5,6}$ or halogenation. ${ }^{7,8}$

Graphite oxide, the most commonly used precursor for graphene synthesis, can be prepared by various methods; with the precise method used having a significant effect on the resulting properties of the final graphene. ${ }^{9}$ The two most common methods are based on the oxidation of graphite in concentrated acids with nitrate and permanganate ions or chlorate ions. Generally, methods based on reaction mixtures with $\mathrm{MnO}_{4}{ }^{-}$

${ }^{a}$ Institute of Chemical Technology, Department of Inorganic Chemistry, 16628 Prague 6, Czech Republic. E-mail: zdenek.sofer@vscht.cz; Fax: +420 22431-0422

${ }^{b}$ Institute of Organic Chemistry and Biochemistry AS CR, v.v.i., Flemingovo nam. 2, 166 10 Prague 6, Czech Republic. E-mail: Stanislava.Matejkova@uochb.cas.cz; Fax: +420 220183578

${ }^{c}$ Division of Chemistry \& Biological Chemistry, School of Physical and Mathematical Sciences, Nanyang Technological University, Singapore, 637371, Singapore. E-mail: pumera@ntu.edu.sg; Fax: +65 6791-1961

$\dagger$ Electronic supplementary information (ESI) available. See DOI: 10.1039/c4nr01154f ions yield graphite oxides with higher concentrations of carboxylic acid functional groups. Materials prepared in the presence of $\mathrm{ClO}_{3}{ }^{-}$ions primarily contain hydroxyl and epoxide functional groups. Such differences provide the basis for the chemical modification of graphite oxide.

The halogen-based chemical modification of graphene and graphite oxides has only been reported by a few authors, most of whom used methods based on the exfoliation of graphite oxide under a halogen atmosphere or on UV-enhanced halogenation with elemental halogens. Fluorinated graphene, fluorographene, has been the most widely studied derivative of the halogenated graphenes. ${ }^{\mathbf{1 0} 11}$ Although this material exhibits many unique properties, its relatively strong $\mathrm{F}-\mathrm{C}$ bond makes it unsuitable for further chemical modification. For this purpose, because of their high reactivity, the most suitable materials are bromine derivatives of graphene. It is, therefore, surprising that few researchers have focused on the synthesis of brominated graphene. ${ }^{\mathbf{1 2 , 1 3}}$ While the structural and electronic properties of $\mathrm{Br}_{2} / \mathrm{Br}$ adsorption and intercalation have been studied ${ }^{\mathbf{1 4}}$ and DFT (density-functional theory) has been applied to a study of graphene bromination, ${ }^{15}$ no systematic complex study has yet been published.

Thus, we present several scalable methods for the synthesis of bromine derivatives of graphene with different amounts of bromine and different concentrations of the remaining functional groups. To optimize this synthesis, graphite oxide was prepared according to either the Hummers ${ }^{\mathbf{1 6}}$ or Hofmann ${ }^{\mathbf{1 7}}$ method. We show the influence of each method on the bromine 
concentrations of the resulting brominated graphene and characterize the synthesized brominated graphenes using an extensive spectrum of analytical techniques.

\section{Experimental}

Graphite oxide (GO) was prepared according to either the Hofmann or Hummers method from high-purity microcrystalline

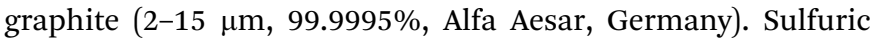
acid $(98 \%)$, nitric acid $(68 \%)$, potassium chlorate $(>99 \%)$, potassium permanganate $(>99.5 \%)$, hydrogen peroxide $(30 \%)$, sodium nitrate $(99.5 \%)$, hydrochloric acid (37\%), hydrobromic acid (48\%), bromine (>99.5\%), iron (>99\%), methanol (>99.9\%) and $N, N$-dimethylformamide (DMF) were obtained from Penta (Czech Republic). Potassium hydrogen phosphate and potassium dihydrogenphosphate were obtained from Lach-Ner (Czech Republic). Argon of 99.996\% purity was obtained from SIAD (Czech Republic). Deionized water (16.8 Mohm) was used for buffer preparation. Nylon and Teflon filtration membranes with a porosity of $0.45 \mu \mathrm{m}$ (Vitrum, Czech Republic) were used for the filtration of the reaction products.

The graphite oxide prepared by the Hofmann method ${ }^{\mathbf{1 7}}$ was termed 'HO-GO'. Concentrated sulfuric acid $(87.5 \mathrm{~mL})$ and nitric acid $(27 \mathrm{~mL})$ were added to a reaction flask containing a magnetic stir bar. The mixture was then cooled at $0{ }^{\circ} \mathrm{C}$, and graphite $(5 \mathrm{~g})$ was added. The mixture was vigorously stirred to avoid agglomeration and to obtain a homogeneous dispersion. While keeping the reaction flask at $0{ }^{\circ} \mathrm{C}$, potassium chlorate (55 g) was slowly added to the mixture in order to avoid a sudden increase in temperature and the consequent formation of explosive chlorine dioxide gas. Upon the complete dissolution of potassium chlorate, the reaction flask was then loosely capped to allow the escape of the evolved gas and the mixture was continuously vigorously stirred for $96 \mathrm{~h}$ at room temperature. On completion of the reaction, the mixture was poured into deionized water ( $3 \mathrm{~L}$ ) and decanted. The graphite oxide was first redispersed in $\mathrm{HCl}(5 \%)$ solutions to remove sulfate ions and then repeatedly centrifuged and redispersed in deionized water until all chloride and sulfate ions were removed. The graphite oxide slurry was then dried in a vacuum oven at $50{ }^{\circ} \mathrm{C}$ for $48 \mathrm{~h}$ before use.

The second graphite oxide, 'HU-GO' was synthesized in a way similar to the Hummers method. ${ }^{16}$ Graphite $(5 \mathrm{~g})$ and sodium nitrate $(2.5 \mathrm{~g})$ were stirred together with concentrated sulfuric acid $(115 \mathrm{~mL})$. The mixture was then cooled at $0{ }^{\circ} \mathrm{C}$. Potassium permanganate $(15 \mathrm{~g}$ ) was then added with vigorous stirring for $2 \mathrm{~h}$. During the following four hours, the reaction mixture was allowed to reach room temperature before being heated to $35^{\circ} \mathrm{C}$ for $30 \mathrm{~min}$. The reaction mixture was then poured into a flask of deionized water $(250 \mathrm{~mL})$ and heated to $70{ }^{\circ} \mathrm{C}$ for $15 \mathrm{~min}$. The mixture was then poured into deionized water $\left(\begin{array}{ll}1 & \mathrm{~L}\end{array}\right)$. The unreacted potassium permanganate and manganese dioxide were removed by the addition of $3 \%$ hydrogen peroxide. The reaction mixture was then allowed to settle before being decanted. The obtained graphite oxide was then purified by repeated centrifugation and redispersion in deionized water until all sulfate ions were removed. The graphite oxide slurry was then dried in a vacuum oven at $50{ }^{\circ} \mathrm{C}$ for $48 \mathrm{~h}$ before use.

The synthesis of brominated graphene was performed for both HO-GO and HU-GO using five different methods.

The first method was based on the reflux of graphite oxide with bromine. Graphite oxide $(250 \mathrm{mg})$ was placed in a $100 \mathrm{~mL}$ flask with a magnetic stirring bar, and bromine $(10 \mathrm{~mL})$ was added. The reaction mixture was heated under reflux with stirring for $5 \mathrm{~h}$. After the reaction mixture was cooled, the unreacted bromine was evaporated at room temperature. The reaction product was washed with deionized water and separated by suction filtration on the nylon membrane and repeatedly washed with deionized water and methanol. The samples prepared according to this procedure were termed ' $\mathrm{HO}-\mathrm{Br}$ ' and 'HU-Br'.

The second method involved the addition of iron powder to the reaction mixture. Graphite oxide $(250 \mathrm{mg})$ was placed in a $100 \mathrm{~mL}$ reaction flask with a magnetic stirring bar, and iron powder $(50 \mathrm{mg})$ was added. Subsequently, bromine $(10 \mathrm{~mL})$ was added and the reaction mixture was refluxed and stirred for $5 \mathrm{~h}$. The remaining bromine was removed from the reaction mixture by vacuum filtration and, to remove iron, the product was washed with deionized water and diluted hydrobromic acid ( $1: 2$ by vol.). Finally, graphene was washed with deionized water and methanol. These samples were termed ' $\mathrm{HO}-\mathrm{Br} / \mathrm{Fe}$ ' and 'HU-Br/Fe'.

The third method involved bromination at atmospheric pressure with hydrobromic acid. Graphite oxide (250 mg) was placed in a $100 \mathrm{~mL}$ reaction flask with a magnetic stirring bar, and hydrobromic acid $(15 \mathrm{~mL})$ was added. The reaction mixture was stirred and refluxed for $5 \mathrm{~h}$. The reaction product was separated by suction filtration, and repeatedly washed with deionized water and methanol. These samples were termed 'HO-HBr' and 'HU-HBr'.

The fourth method of synthesis was performed with bromine at elevated pressure. Graphite oxide $(250 \mathrm{mg})$ and bromine $(10 \mathrm{~mL})$ were placed in a Teflon-lined stainless steel autoclave. The autoclave was heated at $240{ }^{\circ} \mathrm{C}$ for $5 \mathrm{~h}$. The unreacted bromine was evaporated at room temperature and the product was washed from the reaction vessel using deionized water. Next, the product was separated from the suspension by suction filtration on the nylon membrane and repeatedly washed with deionized water. These products were termed 'HO-Br-A' and 'HU-Br-A'.

The fifth method was performed with hydrobromic acid at elevated pressure. Graphite oxide $(250 \mathrm{mg})$ and hydrobromic acid $(10 \mathrm{~mL})$ were placed in the Teflon-lined stainless steel autoclave. The autoclave was heated at $240{ }^{\circ} \mathrm{C}$ for $5 \mathrm{~h}$. The unreacted bromine was evaporated at room temperature and the product was washed from the reaction vessel using deionized water. Next, the reaction mixture was directly filtrated by suction filtration on the nylon membrane and repeatedly washed with deionized water and methanol. These samples were termed 'HO-HBr-A' and 'HU-HBr-A'.

The morphology of the brominated graphenes was investigated using a scanning electron microscope (SEM) with a FEG electron source (Tescan Lyra dual beam microscope). Elemental 
composition and mapping analyses were performed using an energy dispersive spectroscopy (EDS) analyzer $\left(\mathrm{X}-\mathrm{Max}^{\mathrm{N}}\right)$ with a $20 \mathrm{~mm}^{2}$ SDD detector (Oxford instruments) and AZtecEnergy software. To conduct the measurements, the samples were placed on carbon conductive tape. SEM and SEM-EDS measurements were carried out using a $10 \mathrm{kV}$ electron beam.

Combustible elemental analysis (CHNS-O) was performed using a PE 2400 Series II CHNS/O Analyzer (Perkin Elmer, USA). The instrument was used in CHN operating mode (the most robust and interference-free mode) to convert the sample elements to simple gases $\left(\mathrm{CO}_{2}, \mathrm{H}_{2} \mathrm{O}\right.$ and $\left.\mathrm{N}_{2}\right)$. The PE 2400 analyzer automatically performed combustion, reduction, homogenization of product gases, separation and detection. An MX5 microbalance (Mettler Toledo) was used for precise weighing of the samples (1.5-2.5 mg per single sample analysis). Using this procedure, the accuracy of CHN determination was better than $0.30 \%$ abs. Internal calibration was performed using $N$-fenyl urea.

To measure the bromine concentration, the samples were decomposed for analysis according to the Schöniger method. An exact amount of sample ( $c a .10 \mathrm{mg}$ ) was wrapped in ash-free paper, burned under a pure oxygen atmosphere and leached out with deionized water. $\mathrm{The}^{-} \mathrm{Br}^{-}$ions formed were titrated with a solution of $\mathrm{Hg}\left(\mathrm{NO}_{3}\right)_{2}$ using sodium nitroprusside as an indicator.

High resolution X-ray photoelectron spectroscopy (XPS) was performed using an ESCAProbeP spectrometer (Omicron Nanotechnology Ltd, Germany) with a monochromatic aluminum X-ray radiation source $(1486.7 \mathrm{eV})$. Wide-scan surveys of all elements were performed, with subsequent highresolution scans of the $\mathrm{C} 1 \mathrm{~s}, \mathrm{O} 1 \mathrm{~s}, \mathrm{Br} 3 \mathrm{~d}$ and $\mathrm{Br} 3 \mathrm{p}$ peaks. Relative sensitivity factors were used to evaluate the carbon-tooxygen $(\mathrm{C} / \mathrm{O})$ ratios from the survey spectra. The samples were placed in a conductive carrier made from a high-purity silver bar. An electron gun was used to eliminate sample charging during measurement (1-5 V).

An inVia Raman microscope (Renishaw, England) was used for Raman spectroscopy in a backscattering geometry with an ultra-low noise RenCam CCD detector. For the measurements, a Nd-YAG laser was used $(532 \mathrm{~nm})$ with a $50 \times$ magnification objective. Instrument calibration was achieved using a silicon reference which gave the typical peak position at $520 \mathrm{~cm}^{-1}$ and a resolution of less than $1 \mathrm{~cm}^{-1}$. To ensure a sufficiently strong signal and to avoid radiation damage to the samples, the laser power used for these measurements ranged from 0.5 to $50 \mathrm{~mW}$. The samples were suspended in deionized water $\left(1 \mathrm{mg} \mathrm{mL} \mathrm{mL}^{-1}\right)$ and ultrasonicated for $10 \mathrm{~min}$. The suspension was deposited on a small piece of silicon wafer and dried. For high temperature measurements, a TS1500EV high temperature cell (Linkam, England) was used. Measurements were performed with a $20 \times$ magnification long-working-distance objective under an argon atmosphere.

Fourier transform infrared spectroscopy (FTIR) measurements were performed on a NICOLET 6700 FTIR spectrometer (Thermo Scientific, USA). A Diamond ATR crystal and a DTGS detector were used for all measurements, which were carried out in the range $4000-400 \mathrm{~cm}^{-1}$ at a resolution of $2 \mathrm{~cm}^{-1}$.
To measure the electrical resistivity of brominated graphene, $40 \mathrm{mg}$ was compressed into a capsule (1/4 inch diameter) at a pressure of $400 \mathrm{MPa}$ for $30 \mathrm{~s}$. The resistivity of the capsules was measured by a four-point technique using the van der Pauw method. ${ }^{18}$ The resistivity measurements were performed with a Keithley 6220 current source and an Agilent 34970A data acquisition/switch unit. The measuring current was set to $10 \mathrm{~mA}$.

Thermal behavior was analyzed by simultaneous thermal analysis (STA). The DTA and TG curves were recorded simultaneously on a Linseis STA PT1600 apparatus at a heating rate of $10{ }^{\circ} \mathrm{C} \mathrm{min}^{-1}$ from ambient temperature to $1000{ }^{\circ} \mathrm{C}$ under a dynamic oxygen atmosphere $\left(50 \mathrm{~cm}^{3} \mathrm{~min}^{-1}\right)$.

Electrochemical characterization was performed by cyclic voltammetry using an Interface 1000 potentiostat (Gamry, USA) with a three-electrode setup. The glassy carbon working electrode (GC), platinum auxiliary electrode (Pt) and $\mathrm{Ag} / \mathrm{AgCl}$ reference electrode were obtained from Gamry (USA). For the cyclovoltammetric measurements, graphene was dispersed in DMF $\left(1 \mathrm{mg} \mathrm{mL}^{-1}\right)$ and $3 \mu \mathrm{L}$ was evaporated on the glass carbon working electrode. All potentials stated in the following section were measured against the $\mathrm{Ag} / \mathrm{AgCl}$ reference electrode. To investigate the inherent electrochemistry, a phosphate buffer solution (PBS, $50 \mathrm{mM}, \mathrm{pH}=7.2$ ) was used as the supporting electrolyte. The HET rate was measured using a $10 \mathrm{mM}$ $\mathrm{K}_{4}\left[\mathrm{Fe}(\mathrm{CN})_{6}\right] / \mathrm{K}_{3}\left[\mathrm{Fe}(\mathrm{CN})_{6}\right]$ redox probe, with a $50 \mathrm{mM}$ PBS solution as the supporting electrolyte.

\section{Results and discussion}

The Hummers and Hofmann methods were used to prepare two graphite oxides. The graphite oxides were then brominated using the five methods illustrated in Scheme 1 (for more details and terminology, see the Experimental section). All the prepared materials were thoroughly characterized in order to understand the bromination process.

The morphology of all the brominated graphenes was studied by scanning electron microscopy (SEM) at various magnifications. All samples exhibited the platelet structure typical of graphene-based materials, which suggests that none of the various syntheses applied to the starting graphite oxide had a significant effect on their morphology (Fig. 1).

The distribution of bromine, carbon and oxygen in all samples was measured by SEM-EDS. Elemental composition showed that the amount of bromine in the structure differed significantly depending on the method of synthesis used (Table 1). While no bromine was detected in the HO-Br sample, $26 \mathrm{wt} \%$ of bromine was found in $\mathrm{HO}-\mathrm{HBr}$. A high amount of bromine was also determined in $\mathrm{HO}-\mathrm{HBr}-\mathrm{A}$ (20.2 wt\% of $\mathrm{Br}$ ).

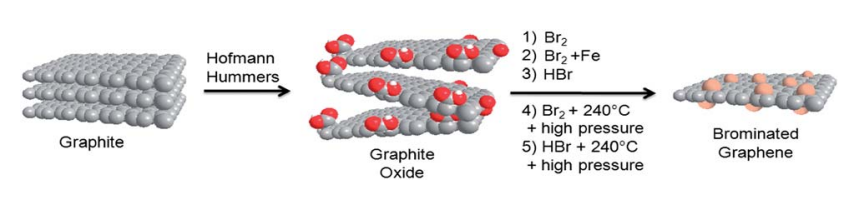

Scheme 1 Synthesis of brominated graphenes. 


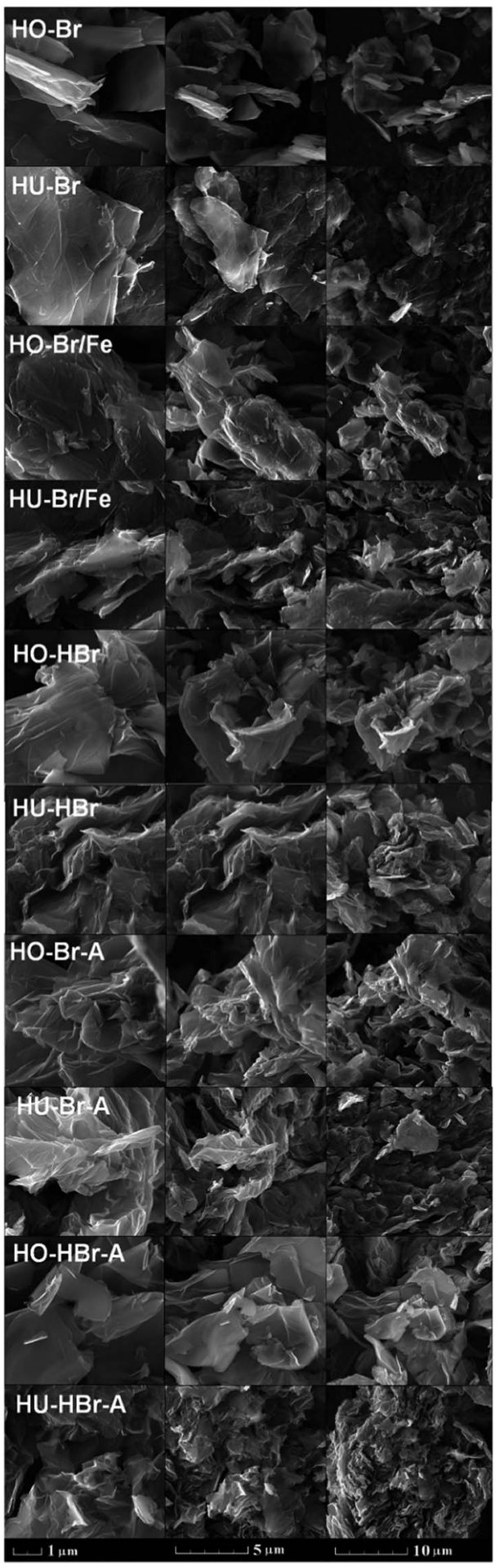

Fig. 1 SEM images of all individual brominated graphenes.

Different C/O ratios were obtained: the concentration of oxygen was very low in the highly brominated samples, but remained high in the less brominated samples. In addition to $\mathrm{C}, \mathrm{O}$ and $\mathrm{Br}$, some impurities were also present. Apart from $\mathrm{S}$ and $\mathrm{Cl}$, some samples also contained $\mathrm{K}, \mathrm{Mn}, \mathrm{Na}$ and $\mathrm{Fe}$ impurities in concentrations lower than $0.2 \mathrm{wt} \%$.
Table 1 Elemental composition of brominated graphenes obtained by SEM-EDS

\begin{tabular}{llrrll}
\hline Sample & wt\% C & wt\% O & wt\% Br & wt\% S & wt\% Cl \\
\hline HO-Br & 73.5 & 25.9 & 0.0 & 0.4 & 0.2 \\
HU-Br & 69.8 & 26.8 & 1.3 & 1.7 & 0.1 \\
HO-Br/Fe & 70.0 & 16.1 & 13.2 & 0.2 & 0.4 \\
HU-Br/Fe & 80.1 & 12.3 & 4.0 & 0.0 & 0.2 \\
HO-HBr & 72.2 & 1.7 & 26.0 & 0.0 & 0.0 \\
HU-HBr & 84.2 & 9.6 & 5.5 & 0.1 & 0.3 \\
HO-Br-A & 85.4 & 8.1 & 5.9 & 0.5 & 0.1 \\
HU-Br-A & 75.8 & 12.9 & 9.5 & 1.5 & 0.1 \\
HO-HBr-A & 78.0 & 1.8 & 20.2 & 0.0 & 0.0 \\
HU-HBr-A & 84.3 & 8.0 & 7.7 & 0.0 & 0.0 \\
\hline
\end{tabular}

The results for element distribution mapping are shown in Fig. 2. Both of the highly brominated samples ( $\mathrm{HO}-\mathrm{HBr}$ and $\mathrm{HO}-$ $\mathrm{HBr}-\mathrm{A})$ showed uniform bromine distribution, indicating that the reaction was performed not only on the edges, but also in the body of the graphite oxide flakes. The composition maps for the samples with lower bromine content are shown in Fig. S1. $\dagger$ In each case, the bromine content of the sample was in agreement with the results obtained for it by the analytical techniques discussed later.

Under very mild reaction conditions (the boiling point of bromine is about $\left.56^{\circ} \mathrm{C}\right)$, either a low concentration $(1.3 \mathrm{wt} \%$ for $\mathrm{HU}-\mathrm{Br}$ ) or practically no concentration (HO-Br) of bromine was detected. This slight difference can be explained by variations in the composition of the oxygen functional groups present in graphite oxide. The most likely reason is that the bromine in HU-Br took the form of a bromine derivative of carboxylic acid, such as carboxyl bromide. Also supporting this assumption is the fact that the bromine content in HU-GO was slightly lower than the concentration of carboxylic acid (measured by alcalimetric titration).

To confirm the results obtained by SEM-EDS and to obtain information on the hydrogen content that SEM-EDS cannot detect, combustible elemental analysis was performed. The results were in very good agreement with those obtained by SEM-EDS (Table 2). The bromine concentrations in the most brominated graphenes were $29.0 \mathrm{wt} \%$ (HO-HBr) and $23.4 \mathrm{wt} \%$ (HO-HBr-A). Compared to SEM-EDS results, the largest deviations were in the oxygen concentrations. This seems to have

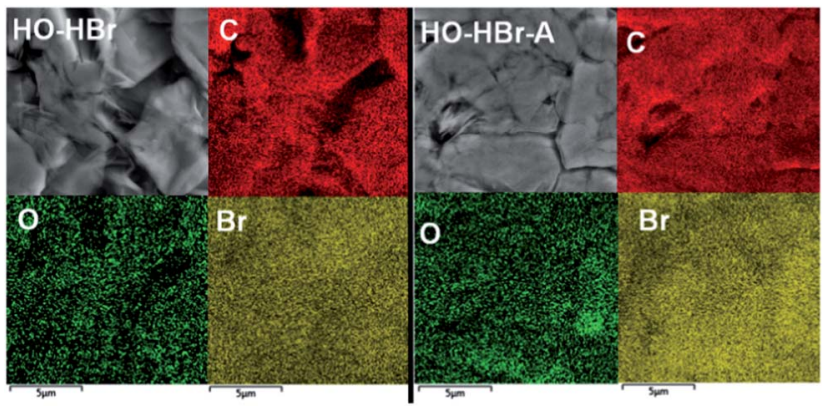

Fig. 2 Element distribution map (SEM-EDS) of highly brominated graphenes $\mathrm{HO}-\mathrm{HBr}$ (left) and $\mathrm{HO}-\mathrm{HBr}-\mathrm{A}$ (right). 
Table 2 Elemental composition of brominated graphenes obtained by elemental combustible analysis

\begin{tabular}{llllrr}
\hline Sample & wt\% C & wt\% H & wt\% N & Wt\% Br & wt\% O \\
\hline HO-Br & 57.35 & 1.65 & 0.00 & 0.00 & 41.00 \\
HU-Br & 51.79 & 2.5 & 0.46 & 3.77 & 41.48 \\
HO-Br/Fe & 58.02 & 0.93 & 0.00 & 20.71 & 20.34 \\
HU-Br/Fe & 56.62 & 2.71 & 2.67 & 3.64 & 34.36 \\
HO-HBr & 68.76 & 0.29 & 0.00 & 28.95 & 2.00 \\
HU-HBr & 81.87 & 0.43 & 0.00 & 7.81 & 9.89 \\
HO-Br-A & 80.75 & 0.35 & 0.00 & 7.11 & 11.79 \\
HU-Br-A & 80.75 & 0.80 & 0.24 & 8.29 & 9.92 \\
HO-HBr-A & 76.06 & 0.09 & 0.00 & 23.41 & 0.44 \\
HU-HBr-A & 82.49 & 0.38 & 0.19 & 10.10 & 6.84 \\
\hline
\end{tabular}

been due to the indirect determination of oxygen by the subtraction of the combined concentrations of $\mathrm{C}, \mathrm{H}, \mathrm{N}$ and $\mathrm{Br}$ from the total concentration $(100 \mathrm{wt} \%)$. The results of elemental analysis in at\% are shown in Table 2.

The three samples with the highest bromine content were the graphenes brominated from HO-GO. As a rule, GO prepared by chlorate oxidation methods contains higher amounts of highly reactive functional groups (e.g. epoxides) compared to GO prepared by permanganate methods (HU-GO). In the case of HO-GO bromination, it seems likely that the opening of the epoxide ring was accompanied by the formation of a C-Br bond. This effect was particularly striking when hydrobromic acid was the bromination agent. Even in high temperature/high pressure bromination, the obtained bromine concentration was higher in HO-HBr-A than in HU-HBr-A. These differences reflect the different mechanisms which occur when different reaction materials are used.

Iron was found to have a significant catalytic effect on the bromination of GO. This is presumably due to the in situ formation of iron(III) bromide, a strong Lewis acid, which, we assume, catalyzed the bromination process according to the Friedel-Crafts reaction mechanism. However, this catalytic effect was observed only for the HO-GO graphenes because the carboxylic acid functional groups present in the HU-GO graphenes negatively influenced bromination.

The high temperature/high pressure samples prepared in the autoclave had high $\mathrm{C} / \mathrm{O}$ ratios. The concentration of oxygen was extremely low in HO-HBr-A, but all HO-GO based graphenes showed a high degree of reduction. A similar trend was observed by XPS and SEM-EDS.

The bromination of GO was not only accompanied by a high degree of reduction, but also led to graphene hydrogenation; the presence of $\mathrm{C}-\mathrm{H}$ bonds being confirmed by FTIR. Another indirect indication of the $\mathrm{C}-\mathrm{H}$ bond is the result of elemental analysis expressed in at\% (see Table 2 and $\mathrm{SA} \dagger$ ). The $\mathrm{C}-\mathrm{H}$ bond formation is indicated by a $\mathrm{H} / \mathrm{O}$ ratio higher than one. The H/O ratio of oxygen functional groups is equal to 1 in the most favorable case of hydroxyl groups, 0.5 for carboxylic acids and 0 in ketones or epoxide groups. By the simple subtraction of the oxygen from hydrogen concentrations, we determined that the lowest concentration of hydrogen in the form of $\mathrm{C}-\mathrm{H}$ bonds was 0.91 and 2.50 at\% in HO-HBr-A and $\mathrm{HO}-\mathrm{HBr}$, respectively. The formation of $\mathrm{C}-\mathrm{H}$ bonds can be explained by the reaction of hydrobromic acid with unsaturated $\mathrm{C}-\mathrm{C}$ bonds under the simultaneous formation of $\mathrm{C}-\mathrm{Br}$ and $\mathrm{C}-\mathrm{H}$ bonds. A relatively high $\mathrm{H} / \mathrm{O}$ ratio was also observed for HU-Br-A. In this case, it is likely that, at elevated temperatures, hydrobromic acid formed by the reaction of bromine with water evolved from the carboxylic groups present in HU-GO reacting with unsaturated $\mathrm{C}-\mathrm{C}$ bonds.

High resolution XPS was used to determine the chemical composition of the surface of the brominated graphenes. The XPS survey spectra are presented in Fig. 3, where Br 3d, Br 3p, C $1 \mathrm{~s}$ and $\mathrm{O} 1 \mathrm{~s}$ peaks are clearly visible. The $\mathrm{Br} 3 \mathrm{~d}$ peak was found at $69.0 \mathrm{eV}$, its twin Br 3p peak at $\sim 182 \mathrm{eV}$ and $\sim 189 \mathrm{eV}$, the C 1s peak at $\sim 284.5 \mathrm{eV}$ and the $\mathrm{O} 1 \mathrm{~s}$ peak at $\sim 533 \mathrm{eV}$. The $\mathrm{Br} 3 \mathrm{~s}$ peak at $\sim 256 \mathrm{eV}$ was weak and was only seen in samples with a high amount of bromine. An F 1s peak was also present in some samples at $689.0 \mathrm{eV}$; although no fluorine was detected by SEMEDS, a small amount of fluorine probably originated from the vacuum grease used in the synthesis procedure. These spectra were used to calculate the concentrations of $\mathrm{C}, \mathrm{O}$ and $\mathrm{Br}$. The bromine concentrations obtained from XPS were lower than those obtained by elemental analysis and SEM-EDS measurements. These differences can be ascribed to the surface sensitivity of XPS and, possibly, also to the partial decomposition of the $\mathrm{C}-\mathrm{Br}$ bond in graphene under UHV conditions. The

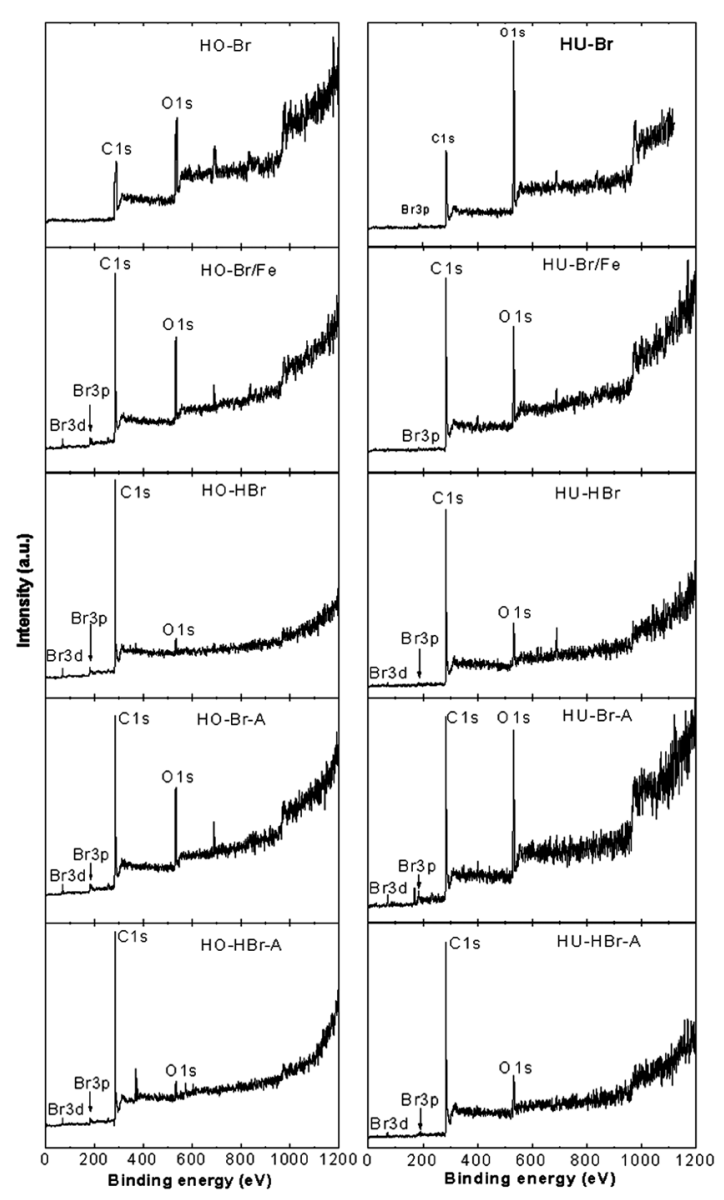

Fig. 3 XPS survey spectra of brominated graphenes. 
obtained concentrations and $\mathrm{C} / \mathrm{O}$ ratios are summarized in Table 3.

The extremely high $\mathrm{C} / \mathrm{O}$ ratios obtained from XPS for graphite oxides reduced by $\mathrm{HBr}$ under reflux ( $\mathrm{HO}-\mathrm{HBr}$ and $\mathrm{HU}-\mathrm{HBr}$ ) or in an autoclave (HO-HBr-A and $\mathrm{HU}-\mathrm{HBr}-\mathrm{A}$ ) are very unusual without thermal reduction. In fact, the $\mathrm{C} / \mathrm{O}$ ratios are comparable to or even higher than those obtained for thermally reduced graphenes. The highest $\mathrm{C} / \mathrm{O}$ ratios were obtained for $\mathrm{HO}-\mathrm{HBr}$ (44.7) and HO-HBr-A (18.5). These results are in good agreement with the chemical analysis results discussed earlier. Thus, the bromination/reduction of graphite oxide can be used for the synthesis of brominated graphene free from oxygen functionalities.

The detailed $\mathrm{Br} 3 \mathrm{~d}, \mathrm{Br} 3 \mathrm{p}$ and $\mathrm{O}$ 1s spectra for $\mathrm{HO}-\mathrm{HBr}$ and HO-HBr-A are shown in Fig. 4. The detailed spectra for the other brominated graphenes are presented in Fig. S2-S4, $\uparrow$ respectively. While only one $\mathrm{Br} 3 \mathrm{~d}$ peak and one $\mathrm{O} 1 \mathrm{~s}$ peak are visible, $\mathrm{Br} 3 \mathrm{p}$ is composed of two peaks, $\mathrm{Br} 3 \mathrm{p}_{3 / 2}$ at $\sim 182 \mathrm{eV}$ and $3 \mathrm{p}_{1 / 2}$ at $\sim 189 \mathrm{eV}$. The Br 3d peaks for $\mathrm{HO}-\mathrm{HBr}$ and $\mathrm{HO}-\mathrm{HBr}-\mathrm{A}$ were fitted to obtain the ratio between chemically and physically bonded

Table 3 Quantitative comparison of detected elements from XPS survey spectra (in at\%) and corresponding $\mathrm{C} / \mathrm{O}$ ratios

\begin{tabular}{llrlr}
\hline Sample & at\% C & at\% & at\% $\mathrm{Br}$ & C/O ratio \\
\hline $\mathrm{HO}-\mathrm{Br}$ & 73.9 & 26.1 & 0.0 & 2.8 \\
$\mathrm{HU}-\mathrm{Br}$ & 77.2 & 20.5 & 2.3 & 3.8 \\
$\mathrm{HO}-\mathrm{Br} / \mathrm{Fe}$ & 85.2 & 13.7 & 1.1 & 6.2 \\
$\mathrm{HU}-\mathrm{Br} / \mathrm{Fe}$ & 83.2 & 16.5 & 0.3 & 5.0 \\
$\mathrm{HO}-\mathrm{HBr}$ & 95.2 & 2.1 & 2.7 & 44.7 \\
$\mathrm{HU}-\mathrm{HBr}$ & 90.1 & 9.4 & 0.5 & 9.6 \\
$\mathrm{HO}-\mathrm{Br}-\mathrm{A}$ & 86.3 & 12.9 & 0.9 & 6.7 \\
$\mathrm{HU}-\mathrm{Br}-\mathrm{A}$ & 77.2 & 20.5 & 2.3 & 3.8 \\
$\mathrm{HO}-\mathrm{HBr}-\mathrm{A}$ & 93.1 & 5.0 & 1.9 & 18.5 \\
HU-HBr-A & 91.8 & 7.4 & 0.8 & 12.4
\end{tabular}
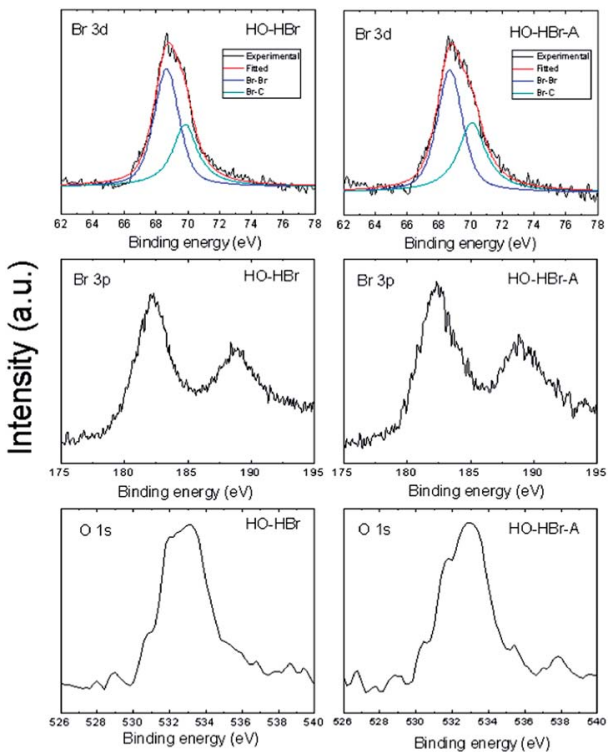

Fig. 4 High-resolution XPS spectra of $\mathrm{Br} 3 \mathrm{~d}, \mathrm{Br} 3 \mathrm{p}$, and $\mathrm{O}$ 1s of $\mathrm{HO}-$ $\mathrm{HBr}$ and $\mathrm{HO}-\mathrm{HBr}-\mathrm{A}$. bromine: a binding energy of $70.0 \mathrm{eV}$ was attributed to the $\mathrm{Br}-\mathrm{C}$ bond and of $68.7 \mathrm{eV}$ to the physically bonded $\mathrm{Br}_{2} \cdot{ }^{19}$ In $\mathrm{HO}-\mathrm{HBr}$ $223 \%$ of bromine was in the form $\mathrm{C}-\mathrm{Br}$ and the remaining bromine was physically sorbed, while in $\mathrm{HO}-\mathrm{HBr}-\mathrm{A} \sim 42 \%$ of bromine was bonded as C-Br (Fig. 4).

A detailed peak-fitting analysis of the $\mathrm{C} 1 \mathrm{~s}$ peak for all brominated graphenes is shown in Fig. 5. High resolution C 1s spectra were fitted to quantitatively differentiate the six different carbon stages: C-C (284.4 eV); C-C/C-H $(285.4 \mathrm{eV})$; C$\mathrm{O} / \mathrm{C}-\mathrm{Br}(286.3 \mathrm{eV}) ; \mathrm{C}=\mathrm{O}(288.0 \mathrm{eV}) ; \mathrm{O}-\mathrm{C}=\mathrm{O}(289.0 \mathrm{eV}) ; \pi-\pi^{*}$ interaction $(290.5 \mathrm{eV})$. The $\mathrm{C}-\mathrm{O}$ and $\mathrm{C}-\mathrm{Br}$ bonds have very similar binding energies: $\mathrm{C}-\mathrm{O}$ has been reported as being $\sim 286.5 \mathrm{eV}$ while the $\mathrm{C}-\mathrm{Br}$ bond was reported as $\sim 286.2 \mathrm{eV} .^{19}$ Hence, an unresolved single peak was observed for both bonds. A similar approach was applied to the $\mathrm{C}-\mathrm{C}$ and $\mathrm{C}-\mathrm{H}$ groups. Significant differences in the composition of these functional groups were found in the brominated graphenes (Table 4). The relatively high concentration of the $\mathrm{C}-\mathrm{H} / \mathrm{C}-\mathrm{C}$ bond in the

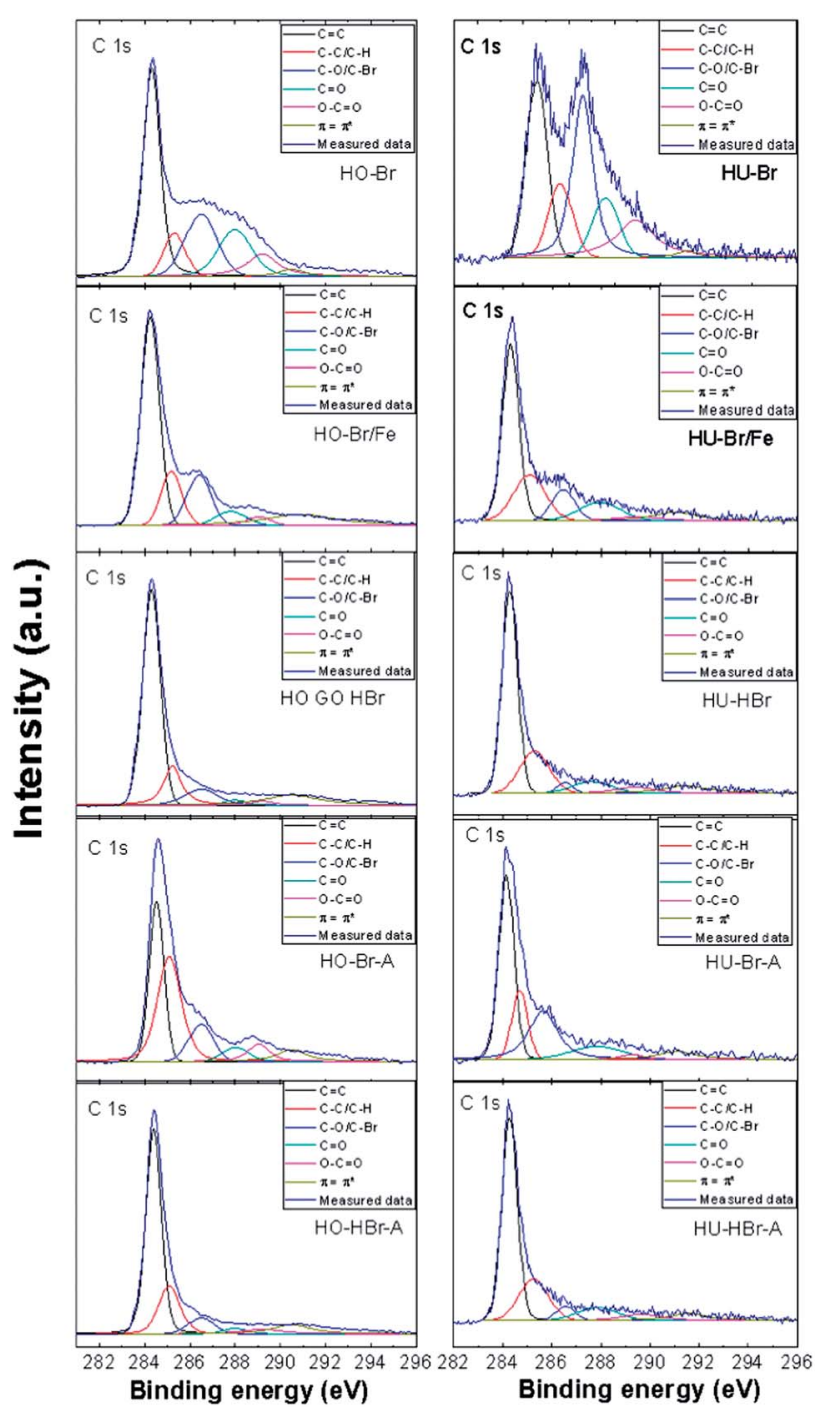

Fig. 5 Details of high resolution XPS spectra of the $C 1$ s peak, where fittings of the individual spectra show the possible carbon bonds. 
Table 4 Quantitative comparison of individual carbon stages in C 1s obtained by high-resolution XPS

\begin{tabular}{lcccrrr}
\hline Sample & $\mathrm{C}=\mathrm{C}$ & $\mathrm{C}-\mathrm{C} / \mathrm{C}-\mathrm{H}$ & $\mathrm{C}-\mathrm{O} / \mathrm{C}-\mathrm{Br}$ & $\mathrm{C}=\mathrm{O}$ & $\mathrm{O}-\mathrm{C}=\mathrm{O}$ & $\pi=\pi^{*}$ \\
\hline HO-Br & 41.0 & 9.1 & 19.8 & 17.3 & 10.1 & 2.7 \\
HU-Br & 44.7 & 21.2 & 10.4 & 11.2 & 4.0 & 8.5 \\
HO-Br/Fe & 48.4 & 13.9 & 14.8 & 5.3 & 2.7 & 14.8 \\
HU-Br/Fe & 27.4 & 13.1 & 30.9 & 11.4 & 15.7 & 1.5 \\
HO-HBr & 58.8 & 14.9 & 9.5 & 2.0 & 2.2 & 12.6 \\
HU-HBr & 53.8 & 21.1 & 2.8 & 8.2 & 5.5 & 8.6 \\
HO-Br-A & 31.5 & 38.0 & 11.9 & 5.2 & 6.6 & 6.8 \\
HU-Br-A & 40.4 & 15.0 & 25.6 & 10.4 & 2.4 & 6.2 \\
HO-HBr-A & 57.8 & 19.5 & 6.9 & 2.5 & 4.7 & 8.5 \\
HU-HBr-A & 54.5 & 20.4 & 4.5 & 8.8 & 5.2 & 6.6 \\
\hline
\end{tabular}

samples synthesized by the reaction with $\mathrm{HBr}$ and $\mathrm{Br}_{2}$ in an autoclave indicates the presence of $\mathrm{C}-\mathrm{H}$ bonds, which was also exhibited by FTIR spectroscopy, Raman spectroscopy and elemental combustion analysis.

To obtain more information about the structure of the synthesized graphenes, their Raman spectra were recorded at a laser power of 5 and $0.5 \mathrm{~mW}$ (Fig. 6 and $55, \dagger$ respectively). Two major bands corresponding to the D-band $\left(1350 \mathrm{~cm}^{-1}\right)$ and G-band $\left(1580 \mathrm{~cm}^{-1}\right)$ were found in all spectra. ${ }^{20}$ The presence of the D-band indicates defects in the graphene layer, primarily

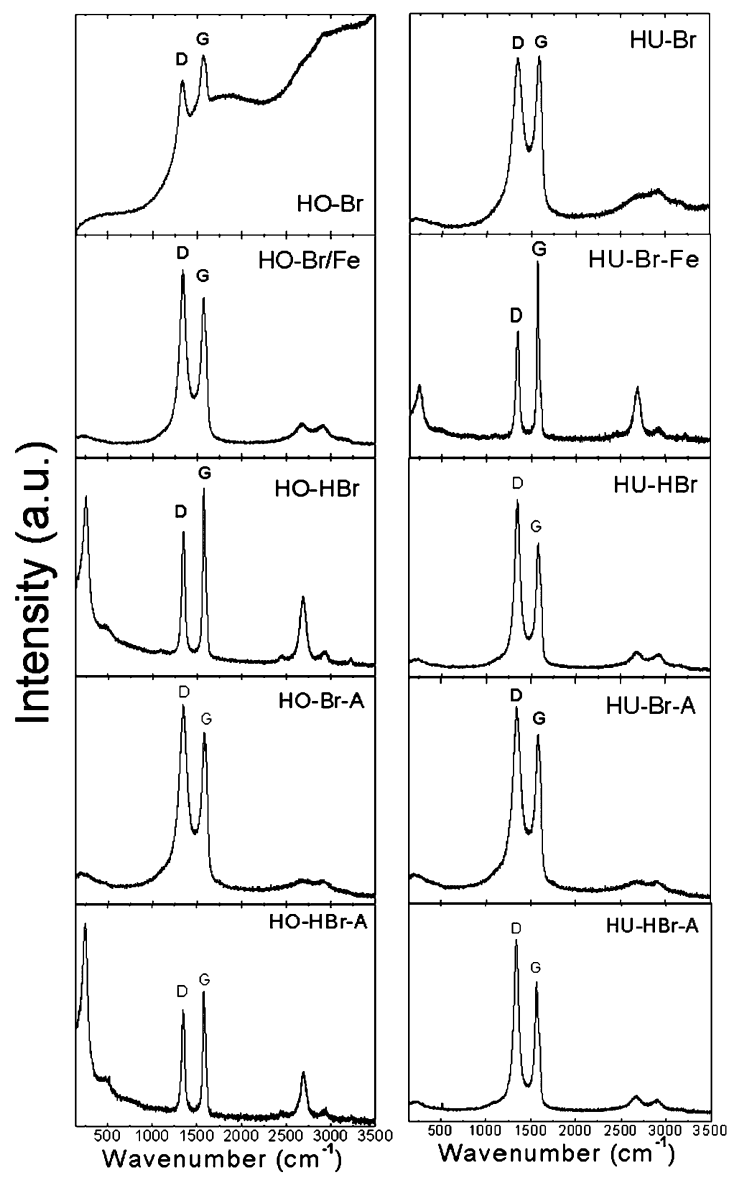

Fig. 6 Raman spectra of brominated graphenes ( $5 \mathrm{~mW}$ laser power). attributable to the presence of $\mathrm{sp}^{3}$ bonded carbon atoms. The G-band resulted from $\mathrm{sp}^{2}$ bonded carbon atoms in the graphene layer. Two other minor bands were located at higher wavenumbers: a 2D band at $2690 \mathrm{~cm}^{-1}$ and a $\mathrm{D}^{\prime \prime}$ band at $2920 \mathrm{~cm}^{-1}$. $^{21}$

The debromination as the reverse process to $\mathrm{C}-\mathrm{Br}$ bond formation was demonstrated by the Raman spectra measurements. ${ }^{22}$ Compared to the Raman spectra recorded at $0.5 \mathrm{~mW}$, the increased laser power of $5.0 \mathrm{~mW}$ led to local heating of brominated graphenes and a decrease in $\mathrm{C}-\mathrm{Br}$ band intensity. This clearly shows the removal of bromine at elevated temperatures and the recovery of the graphene structure.

In the highly brominated samples, additional bands indicating the presence of a C-Br bond were observed at $240 \mathrm{~cm}^{-1}$ and $480 \mathrm{~cm}^{-1}$. Significant differences can be seen between the spectra of HO-GO reacted with bromine under reflux (HO-Br) and all other spectra. In line with the results presented, no significant reduction occurred for $\mathrm{HO}-\mathrm{Br}$, and the high intense luminescence background typical of graphite oxide was observed.

In addition, we calculated the defect-free domains within graphene represented by the value of the average crystallite size $\left(L_{\mathrm{a}}\right)$ according to the equation: ${ }^{23}$

$$
L_{\mathrm{a}}=2.4 \times 10^{-10} \times \lambda_{\text {laser }}^{4} \times I_{\mathrm{G}} / I_{\mathrm{D}}
$$

where $I_{\mathrm{G}} / I_{\mathrm{D}}$ is the ratio of the intensities of the $\mathrm{D}$ and $\mathrm{G}$ bands, respectively, and $\lambda_{\text {laser }}$ refers to the excitation wavelength $(\mathrm{nm})$ used in the measurement of the Raman spectrum (i.e. $\lambda_{\text {laser }}=$ $532 \mathrm{~nm}$ ). Both sets of data ( 0.5 and $5 \mathrm{~mW}$ laser power) were used for the calculation. The results show the strong dependence of D/G ratio and crystallite size on the laser power used (Table 5).

Considering these data, we performed high temperature Raman measurements on the highly brominated graphene HO$\mathrm{HBr}-\mathrm{A}$ to evaluate the influence of temperature on the Raman spectra and C-Br bond stability (Fig. 7). Fig. 7A clearly shows that at higher measurement temperatures a low bromine content was observed, the intensities of the bands at both $240 \mathrm{~cm}^{-1}$ and $480 \mathrm{~cm}^{-1}$ being significantly lower. Also, the D and $\mathrm{G}$ bands shifted to lower wavenumbers as the temperature increased (Fig. 7B). Moreover, the $\mathrm{D} / \mathrm{G}$ intensity ratio was

Table 5 D/G ratios and corresponding crystallite sizes measured by Raman spectroscopy with laser powers of 0.5 and $5 \mathrm{~mW}$

\begin{tabular}{lllll}
\hline Sample & $\begin{array}{l}\mathrm{D} / \mathrm{G} \\
(0.5 \mathrm{~mW})\end{array}$ & $\begin{array}{l}\mathrm{D} / \mathrm{G} \\
(5 \mathrm{~mW})\end{array}$ & $\begin{array}{l}L_{\mathrm{a}}(\mathrm{nm}) \\
(0.5 \mathrm{~mW})\end{array}$ & $\begin{array}{l}L_{\mathrm{a}}(\mathrm{nm}) \\
(5 \mathrm{~mW})\end{array}$ \\
\hline HO-Br & 0.84 & 0.90 & 22.9 & 21.4 \\
HU-Br & 0.96 & 0.99 & 20.0 & 19.4 \\
HO-Br/Fe & 1.30 & 1.17 & 14.8 & 16.4 \\
HU-Br/Fe & 1.06 & 0.66 & 18.1 & 29.1 \\
HO-HBr & 1.20 & 0.77 & 16.0 & 25.0 \\
HU-HBr & 1.25 & 1.32 & 15.4 & 14.6 \\
HO-Br-A & 1.30 & 1.25 & 14.8 & 15.4 \\
HU-Br-A & 1.16 & 1.16 & 16.6 & 16.6 \\
HO-HBr-A & 0.99 & 0.88 & 19.4 & 21.8 \\
HU-HBr-A & 1.17 & 1.29 & 16.4 & 14.9
\end{tabular}



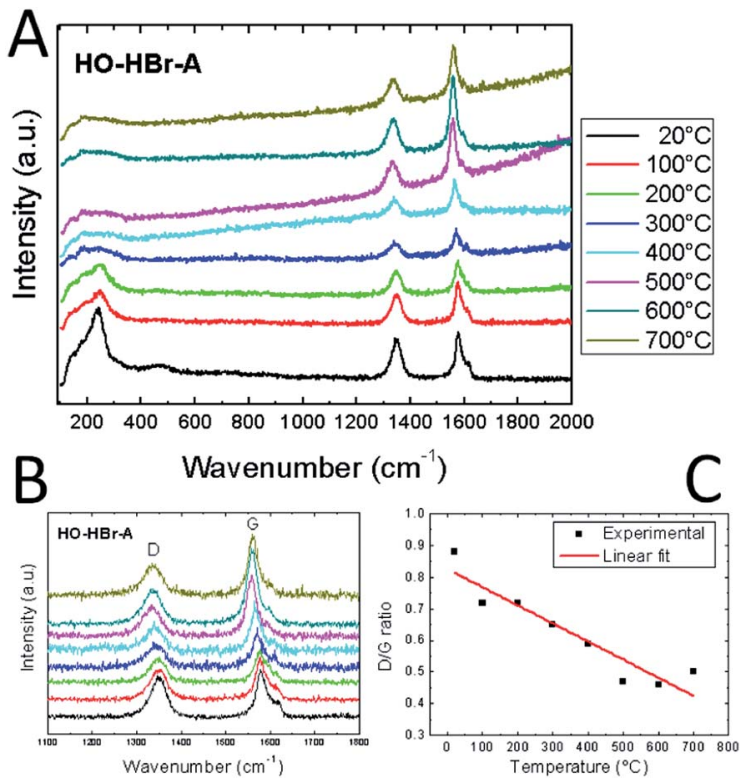

Fig. 7 High temperature Raman measurements of $\mathrm{HO}-\mathrm{HBr}-\mathrm{A}(\mathrm{A})$. Details of $D$ and $G$ bands with a subtracted background $(B)$ and $D / G$ ratios at various temperatures.

significantly lower at higher temperatures. The continual decrease of $\mathrm{D} / \mathrm{G}$ ratio is together with the decrease of $\mathrm{C}-\mathrm{Br}$ vibration band intensity, evidence for continual removal of bromine at elevated temperatures. Breaking of $\mathrm{C}-\mathrm{Br}$ bonds led to restoring of the $\mathrm{sp}^{2}$ hybridized carbon atom layer. This effect is clearly documented in Fig. 7C. At low temperature, the $\mathrm{D}^{\prime}$ band at $1620 \mathrm{~cm}^{-1}$ can be observed as an evident shoulder. This band is frequently attributed to the out/plane vibration related to $\mathrm{sp}^{3}$ hybridized carbon atoms. The decrease of its intensity at elevated temperatures clearly indicates continual removal of bromine at elevated temperatures.

FTIR measurements were used to confirm the formation of the $\mathrm{C}-\mathrm{Br}$ bond and to evaluate the degree of reduction. The high absorption coefficient of graphene represents a serious problem for FTIR and normally only spectra of weak quality are obtained. However, the FTIR spectra for $\mathrm{HO}-\mathrm{HBr}$ and $\mathrm{HO}-\mathrm{HBr}-\mathrm{A}$ clearly show the presence of $\mathrm{C}-\mathrm{Br}$ and $\mathrm{C}-\mathrm{H}$ bonds (Fig. 8). In addition to the $\mathrm{C}-\mathrm{Br}$ band found at $470 \mathrm{~cm}^{-1}$, the remaining oxygen functionalities result in the presence of a broad band at 1050$1200 \mathrm{~cm}^{-1}$ attributed to C-O vibration modes. The characteristic vibration of a graphene layer is located at $1590 \mathrm{~cm}^{-1}$. The
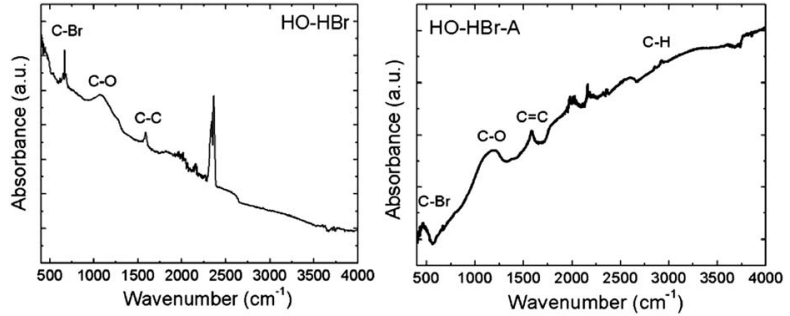

Fig. 8 FTIR spectra of $\mathrm{HO}-\mathrm{HBr}$ and $\mathrm{HO}-\mathrm{HBr}-\mathrm{A}$. two small relatively narrow bands at $2850 \mathrm{~cm}^{-1}$ and $2920 \mathrm{~cm}^{-1}$ reveal the formation of the $\mathrm{C}-\mathrm{H}$ bond during the reaction with hydrobromic acid or bromine under high pressure/high temperature conditions.

The electrical resistivity of brominated graphenes was measured using the four-point method. Significant differences were observed between the samples. The resistivity values were scaled to the concentration of the remaining oxygen functionalities and were not highly affected by the bromine concentration (Table $\mathrm{SB} \dagger$ ). The lowest resistivity values were obtained for the samples with the highest degree of reduction (i.e. samples reduced with $\mathrm{HBr}$ under reflux or in a autoclave). These samples exhibited resistivities ranging from $6.6 \times 10^{-4} \Omega \mathrm{cm}$ (HO-HBr) to $5.1 \times 10^{-3} \Omega \mathrm{cm}$ (HO-HBr-A). HU-GO brominated graphenes displayed higher resistivity, most likely because of the higher concentration of $\mathrm{C}=\mathrm{O}$ functional groups on the parent graphite oxide. Thus, the main factor influencing sample resistivity seems to be the concentration of the remaining oxygen functional groups.

The thermal stability of brominated graphenes was investigated under a dynamic oxygen atmosphere. STA measurements revealed an exothermic peak located around $200{ }^{\circ} \mathrm{C}$ for samples with a high concentration of oxygen functional groups (Fig. 9). At this temperature, the removal of oxygen functionalities led to
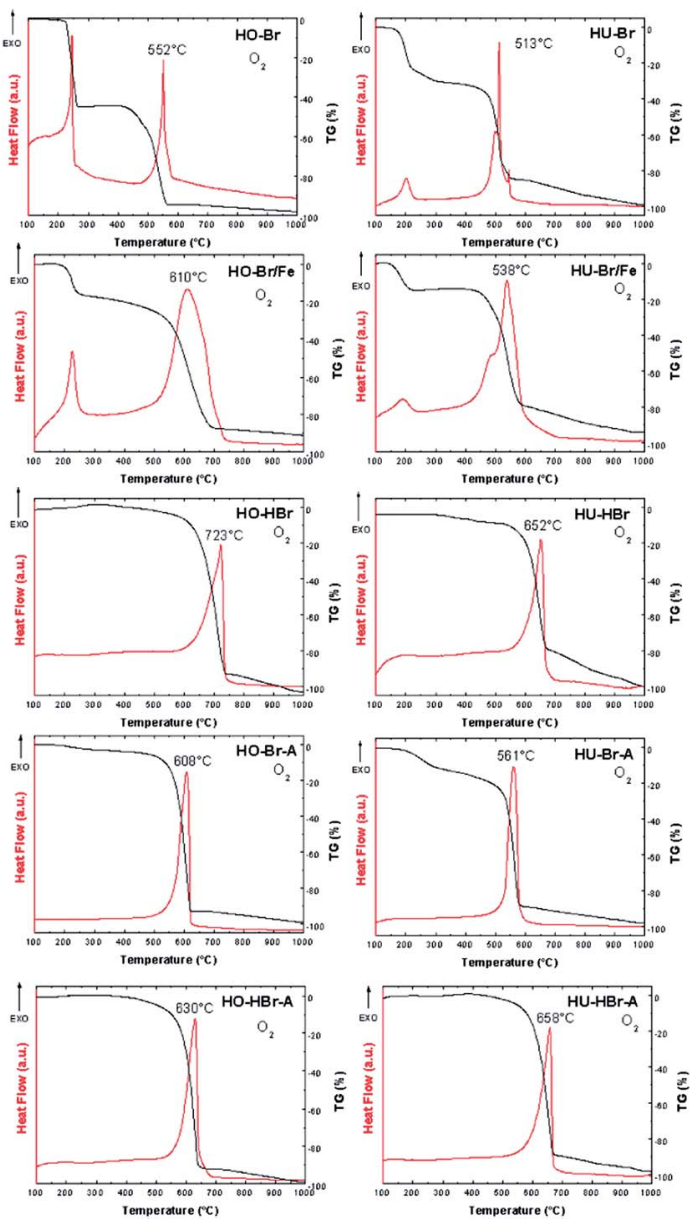

Fig. 9 STA measurements of brominated graphenes. 
weight reduction. Fig. 9 also shows high differences between the onset temperatures for the oxidation-combustion of brominated graphenes. As the bromine concentration increased, graphene became more stable against combustion. The combustion temperature increased by $170{ }^{\circ} \mathrm{C}$ for the most brominated graphene, $\mathrm{HO}-\mathrm{HBr}$ ( $26 \mathrm{wt} \% \mathrm{Br}$ by SEM-EDS).

To obtain more information about the inherent electrochemistry and behavior of the remaining oxygen functional groups and physically sorbed bromine, cyclic voltammetry was performed in a $50 \mathrm{mM}$ PBS solution $(\mathrm{pH}=7)$ in the range of +1.0 to $-1.5 \mathrm{~V}$ (Fig. S6 $\dagger$ ). ${ }^{24}$ The HU-Br and HO-Br samples showed the most distinctive inherent electrochemical behavior. These samples remained almost unchanged, with most of the oxygen functionalities remaining on the graphene surface. In the case of $\mathrm{HO}-\mathrm{Br}$, the reduction of oxygen functionalities started at $-0.50 \mathrm{~V}$ and reached its maximum at $-1.26 \mathrm{~V}$. The electrochemical reduction of HU-Br took place in two steps, with the first maximum at $-0.42 \mathrm{~V}$ and the second at $-1.38 \mathrm{~V}$. The reduction of the remaining oxygen functionalities was observed for $\mathrm{HU}-\mathrm{Br} / \mathrm{Fe}$ and $\mathrm{HO}-\mathrm{Br} / \mathrm{Fe}$, starting at $-0.20 \mathrm{~V}$ and $-0.34 \mathrm{~V}$, respectively. The oxidation at $0.85 \mathrm{~V}$ observed for $\mathrm{HO}-\mathrm{Br} / \mathrm{Fe}$ resulted from the traces of iron impurities. The inherent electrochemical properties of HO-Br-A and HU-Br-A differed due to their lower concentration of remaining oxygen functionalities. In HU-Br-A sample reduction started at $-0.31 \mathrm{~V}$ and proceeded until $-1.5 \mathrm{~V}$ without any significant maxima. The reduction of HO-Br-A started at just $0.03 \mathrm{~V}$ and reached its maximum at $-0.63 \mathrm{~V}$. The significant peak at $+0.25 \mathrm{~V}$ was most probably caused by the oxidation of previously reduced physically sorbed bromine on the surface of the graphene. The most reduced samples, those synthesized by the reaction of graphite oxide with hydrobromic acid, showed reduction peaks between -0.5 $\mathrm{V}$ and $-1.0 \mathrm{~V}(-0.91 \mathrm{~V},-1.07 \mathrm{~V},-0.66 \mathrm{~V}$, and $-0.74 \mathrm{~V}$ for HO$\mathrm{HBr}-\mathrm{A}$, HO-HBr, HU-HBr-A and $\mathrm{HU}-\mathrm{HBr}$, respectively). The inherent electrochemistry of the samples was due to the physically sorbed bromine that remained on their surfaces. The different positions of maxima obtained for bromine reduction reflect the significant differences in their electrochemical activity. However, HO-Br and HU-Br/HO-Br-A show that the key factor that determines their inherent electrochemical behavior is not the bromine content, but the reduction of the remaining oxygen functional groups. The current originating from these reduction processes was two orders of magnitude higher than the signal of the samples with low oxygen content.

Further electrochemical characterization was performed using a ferro/ferricyanide electrochemical probe to determine the HET rates (Table 6); HET values were calculated using Nicholson's method. ${ }^{25}$ Large differences in peak-to-peak separation were observed between the HO-GO and HU-GO brominated graphenes (see Fig. 10). Overall, a slower HET rate was observed for the samples brominated in an autoclave compared to those brominated under reflux. Thus, the HET rate is dependent on various factors, such as the structure and defect concentration, which includes the concentration of bromine and remaining oxygen groups. The higher HET rates observed for the samples prepared under reflux suggest that the defect concentration is the most crucial factor in determining the HET
Table 6 Peak-to-peak separation and HET rate for the brominated graphene samples

\begin{tabular}{llr}
\hline Sample & Peak separation $(\mathrm{mV})$ & HET $\left(\mathrm{cm}^{-1}\right)$ \\
\hline HO-Br & 241 & $6.19 \times 10^{-4}$ \\
HU-Br & 148 & $1.67 \times 10^{-3}$ \\
HO-Br/Fe & 158 & $1.95 \times 10^{-3}$ \\
HU-Br/Fe & 486 & $2.22 \times 10^{-5}$ \\
HO-HBr & 207 & $9.82 \times 10^{-4}$ \\
HU-HBr & 228 & $7.38 \times 10^{-4}$ \\
HO-Br-A & 368 & $1.1 \times 10^{-4}$ \\
HU-Br-A & 258 & $4.91 \times 10^{-4}$ \\
HO-HBr-A & 476 & $2.55 \times 10^{-5}$ \\
HU-HBr-A & 711 & $1.05 \times 10^{-6}$ \\
GC electrode & 718 & $9.53 \times 10^{-7}$
\end{tabular}
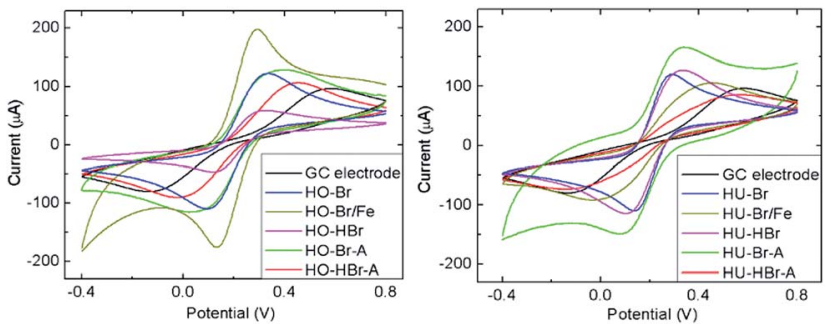

Fig. 10 Electrochemical characterization of brominated graphenes.

rate. However, no significant correlation was observed between the HET rate and the bromine concentration.

\section{Conclusions}

We have successfully prepared brominated graphenes with variable bromine contents by using different graphite oxides, different sources of bromine and different reaction conditions. Brominated graphenes displayed significant differences in bromine concentration, depending on whether they were prepared by the Hummers or Hofmann method. Highly reactive graphite oxides prepared with chlorate-based methods (Hofmann) generally yielded graphenes with higher bromine concentrations than their Hummers equivalents, particularly when $\mathrm{HBr}$ was used as the bromination agent. In the most brominated graphene, $\mathrm{HO}-\mathrm{HBr}-\mathrm{A}$, the bromine concentration exceeded $26 \mathrm{wt} \%$. Bromination using hydrobromic acid was accompanied by the removal of the majority of oxygen functionalities, thereby resulting in a $\mathrm{C} / \mathrm{O}$ ratio higher than 40 . The reactions with hydrobromic acid were also connected with the partial hydrogenation of graphene. STA showed the thermal stability of the brominated graphenes. High temperature Raman spectroscopy confirmed the reverse process of debromination and the recovery of the graphene structure at elevated temperatures. Therefore, the presented brominated graphenes are highly promising starting materials for the synthesis of other graphene derivatives using highly reactive $\mathrm{C}-\mathrm{Br}$ bonds. The reactive $\mathrm{C}-\mathrm{Br}$ bonds can be also used for the development of new biosensors with DNA labeled graphene. Brominated 
graphene can be used in the future for reversible bromine storage. Brominated organics used as flame retardants represent the largest commercial application of bromine. However, due to growing awareness and concerns about their bioaccumulation and various side-effects on the living organisms, a legislation has been enforced in many countries to restrict or even ban the production of some of them (such as PBBs, BDE, etc.). It would be thus highly desirable to find a biologically compatible or at least less toxic substitute. Brominated graphene exhibits several chemical similarities to commercially used brominated flame retardants and it could be thus a potential candidate for their replacement. It also has great potential in the field of electrochemical sensors and microelectronic devices.

\section{Acknowledgements}

This research was supported by a Specific University Research grant, MSMT no. 20/2014. M.P. acknowledges a Tier 2 grant (MOE2013-T2-1-056; ARC 35/13) from the Ministry of Education, Singapore.

\section{Notes and references}

1 A. K. Geim and K. S. Novoselov, Nat. Mater., 2007, 6, 183.

2 P. Blake, P. D. Brimicombe, R. R. Nair, T. J. Booth, D. Jiang, F. Schedin, L. A. Ponomarenko, S. V. Morozov, H. F. Gleeson, E. W. Hill, A. K. Geim and K. S. Novoselov, Nano Lett., 2008, 8, 1704.

3 M. Pumera, A. Ambrosi, A. Bonanni, E. L. K. Chng and H. L. Poh, Trends Anal. Chem., 2010, 29, 954.

4 M. Pumera, Energy Environ. Sci., 2011, 4, 668.

5 D. C. Elias, R. R. Nair, T. M. G. Mohiuddin, S. V. Morozov, P. Blake, M. P. Halsall, A. C. Ferrari, D. W. Boukhvalov, M. I. Katsnelson, A. K. Geim and K. S. Novoselov, Science, 2009, 323, 610.

6 Z. Sofer, O. Jankovský, P. Šimek, L. Soferova, D. Sedmidubsky and M. Pumera, Nanoscale, 2014, 6, 2153.

7 Ch. K. Chua and M. Pumera, J. Mater. Chem., 2012, 22, 23227.
8 O. Jankovský, P. Šimek, D. Sedmidubský, S. Matějková, Z. Janoušek, F. Šembera, M. Pumera and Z. Sofer, RSC Adv., 2014, 4, 1378.

9 H. L. Poh, F. Sanek, A. Ambrosi, G. Zhao, Z. Sofer and M. Pumera, Nanoscale, 2012, 4, 3515.

10 R. R. Nair, W. Ren, R. Jalil, I. Riaz, V. G. Kravets, L. Britnell, P. Blake, F. Schedin, A. S. Mayorov, S. Yuan, M. I. Katsnelson, H.-M. Cheng, W. Strupinski, L. G. Bulusheva, A. V. Okotrub, I. V. Grigorieva, A. N. Grigorenko, K. S. Novoselov and A. K. Geim, Small, 2010, 6, 2877.

11 K. J. Jeon, Z. Lee, E. Pollak, L. Moreschini, A. Bostwick, C. M. Park, R. Mendelsberg, V. Radmilovic, R. Kostecki and T. J. Richardson, ACS Nano, 2011, 5, 1042.

12 H. L. Poh, P. Šimek, Z. Sofer and M. Pumera, Chem.-Eur. J., 2013, 19, 2655.

13 K. Gopalakrishnan, K. S. Subrahmanyam, P. Kumar, A. Govindaraj and C. N. R. Rao, RSC Adv., 2012, 2, 1605.

14 X. Fan, L. Liu, J.-L. Kuo and Z. Shen, J. Phys. Chem. C, 2010, 114, 14939.

15 A. Yaya, C. P. Ewels, I. Suarez-Martinez, Ph. Wagner, S. Lefrant, A. Okotrub, L. Bulusheva and P. R. Briddon, Phys. Rev. B: Condens. Matter Mater. Phys., 2011, 83, 045411.

16 W. S. Hummers and R. E. Offeman, J. Am. Chem. Soc., 1958, 80, 1339.

17 U. Hofmann and A. Frenzel, Kolloid-Z., 1934, 68, 149.

18 L. J. Van der Pauw, Philips Res. Rep., 1958, 13, 1.

19 J. Zheng, H.-T. Liu, B. Wu, Ch.-A. Di, Y.-L. Guo, T. Wu, G. Yu, Y.-Q. Liu and D.-B. Zhu, Sci. Rep., 2012, 2, 662.

20 D. R. Dreyer, R. S. Ruoff and C. W. Bielawski, Angew. Chem., Int. Ed., 2010, 49, 9336.

21 M. S. Dresselhaus, A. Jorio, M. Hofmann, G. Dresselhaus and R. Saito, Nano Lett., 2010, 10, 751.

22 H. L. Poh, P. Simek, Z. Sofer and M. Pumera, Chem.-Eur. J., 2013, 19, 2655.

23 L. G. Cançado, K. Takai, T. Enoki, M. Endo, Y. A. Kim, H. Mizusaki, A. Jorio, L. N. Coelho, R. Magalhães-Paniago and M. A. Pimenta, Appl. Phys. Lett., 2006, 88, 163106.

24 E. L. K. Chng and M. Pumera, Chem.-Asian J., 2011, 6, 2899. 25 R. S. Nicholson, Anal. Chem., 1965, 37, 1351. 